\title{
Two Level Authentication using Biometrics based on Earth Mover's Distance (EMD)
}

\author{
B.Srinivas \\ CSE Department, \\ MVGR College of \\ Engineering, \\ Vizianagaram, AP, India.
}

\author{
U.Neelima \\ CSE Department, \\ MVGR College of \\ Engineering, \\ Vizianagaram, AP, India.
}

\author{
P.Satheesh \\ CSE Department, \\ MVGR College of \\ Engineering, \\ Vizianagaram, AP, India.
}

\author{
Koduganti Venkata \\ Rao \\ Head of the Department, \\ CSE Department, \\ Vignan IIT \\ Vishakanatnam. India.
}

\begin{abstract}
A biometric system which uses a single trait is unable to meet the required security levels. Authentication based on multibiometrics is an emerging trend these days. This paper deals with the biometric authentication system which integrates face and voice biometrics. The system requests the user for an audio sample and an image of his face. The fusion of face biometric with the voice provides a much higher level of certainty a user's identity.We are using the Earth Mover's Distance(EMD), an efficient algorithm for face recognition and comparison of the audio and facial features[15, 16]. The proposed system outwits the single biometric systems by performing two-level authentication and improves system performance. Experimental results were found to have better impact on the system performance.
\end{abstract}

\section{General Terms}

Security, Authentication, biometric.

\section{Keywords}

biometric system, system performance, Authentication, recognition, EMD.

\section{INTRODUCTION}

The concept of identity recognition and the demand for reliable automatic client identity recognition systems for secure accesses had been receiving a lot of attention. The traditional methods involving ID cards or PIN numbers(passwords) have a certain number of drawbacks like Passwords may be forgotten, cards may be lost or stolen and the system cannot effectively differentiate between a user and an bot. Biometrics are primarily driven by the reliability and convenience they provide over classical authentication methods. Biometrics is the process of identifying a person based on his/her physiological or behavioral traits. Most biometric systems deployed in real-world applications depend on a single instance information for authentication.

Each biometric technology has its own pros and cons and no single technology is expected to effectively satisfy the requirements of verification. Some of the drawbacks with unimodal systems can be overcome by integrating two or more biometric systems .Multi-biometric systems, which inherits information from multiple biometric traits , are gaining attention since they are able to solve the problems such as non-universality, noisy sensor data, and spoof attacks which are commonly encountered in unimodal systems.

This authentication approach is promising yet equally challenging in providing robustness and higher accuracy. A biometric-based authentication system consists of two modes; Enrollment and Authentication. In the enrollment mode, the again inputs the biometric data and it is compared with the stored data in database either to identify or verify the user.

In this paper, we introduce a multimodal biometric system which integrates voice and face traits. Face recognition is done by systematically analyzing specific features that are common to everyone's face - the distance between the eyes, width of the nose, position of cheekbones, jaw line, chin and so forth $[1,4]$. These numerical quantities are then combined in a single code that uniquely identifies each person.

The voice sample taken from the user is converted into a set of mathematical values using (MFCC) mel frequency cepstral coefficients algorithm and processed for comparison. Fusion of face and voice gained user acceptability and with largely available cameras and microphones on PCs, user recognition has become a natural solution.

The Earth Mover's Distance (EMD) has emerged as an efficient methodology in object recognition as well as for feature comparison. EMD is a method to evaluate distance between two multi-dimensional distributions in some feature space where a distance measure between single features, which we refer to ground distance is given [8]. EMD computation is based on the solution to well-known transportation problem. It is more robust than histogram techniques and when used to compare distributions with the same mass, the EMD is a true metric.

\section{RELATED WORKS}

This section gives an overview of the related work that has been done for securing a person's identity. Any software being developed must provide security to its legitimate users. The basic idea is to have a two-step authentication process using multimodal biometrics-face and speech based on one of the most efficient algorithms Earth Mover Distance (EMD).The earth mover's distance (EMD) is a measure of the distance between two distributions, and it has been widely used in content-based image retrieval systems, where color images are retrieved from multimedia databases, it is important to apply improved distance measures.

Some derivation algorithms of the EMD have been proposed The Earth Mover's Distance (EMD) is proposed by Rubner et al.[15] and Rubner and Tomasi [14] to compare distributions for image retrieval tasks. The comparison of these distributions was termed as transportation problem (TP).This was solved by an efficient linear algorithm, the transportation simplex (TS) algorithm which solved the problem of EMD. Cohen and Guibas [16] studied the problem of computing a transformation between distributions with minimum EMD. 
Indyk and Thaper [10] proposed a fast approximation EMD algorithm and used it for image retrieval .Unlike the above previous work, we focus on designing an efficient mechanism of person identification using EMD by solving transportation problem and we will show that the proposed EMD comparison achieves better performance and accuracy rates.

\subsection{Pitfalls of Existing system}

Most of the applications use traditional methods of recognizing a person which is password security. This technique has many limitations which includes overhead of remembrance, losing keys, identical passwords for diverse sites etc...these can be easily detected. Hence increasing demand of enhanced security systems has led to an unprecedented interest in biometric based person authentication system. It eliminates problems caused by lost IDs or forgotten passwords by using physiological attributes. These techniques are receiving a lot of attention despite of very few limitations. Biometrics encompasses many techniques out of which face, voice, iris, hand are proving to effectively at their best. It reduces fraud by employing hardto-forge technologies. Despite of these advantages it has been hindered due to many reasons. It provides comparatively less than desirable accuracy in several application domains and these are vulnerable to spoofing attacks. To overcome these shortcomings we use a combination of biometric modalities called multimodal biometrics in an identification system. It enhances reliability and security of the system.

There are certain limitations in unimodal biometric systems. Table 1.1 shows various biometric systems which do false acceptance rate and false reject rates.

Table 1.1 Biometric false acceptance and reject rate.

\begin{tabular}{|c|c|c|}
\hline Biometric & $\begin{array}{c}\text { False Acceptance } \\
\text { Rate }\end{array}$ & $\begin{array}{c}\text { False Reject } \\
\text { Rate }\end{array}$ \\
\hline Fingerprint & $2 \%$ & $2 \%$ \\
\hline Face & $10 \%$ & $1 \%$ \\
\hline Voice & $10-20 \%$ & $2-5 \%$ \\
\hline
\end{tabular}

To overcome this problem we combine two biometric systems such as face recognition and voice to minimize the false acceptance rate and false reject rate.

\section{PROPOSED SYSTEM}

A secure and robust system with a server is designed which is capable enough to make secured authentication using face detection, features extraction and feature comparison based on Earth movers distance algorithm(EMD) [5].

Whenever a user enrolls himself in order to access a particular software application, he must submit his face as an image using the system webcam and recorded speech which would be his password, which are stored in database [11]. The user input voice sample is embedded into face image which in turn is used as a key for authentication. During the phase of verifying user's identity, he needs to submit the key and his facial image as inputs to the system. Now the features are separated from the key and compared with feature sets in database respectively. Next, his facial features are compared with the related feature sets in database using EMD algorithm Based on the threshold value the user's identity is determined.

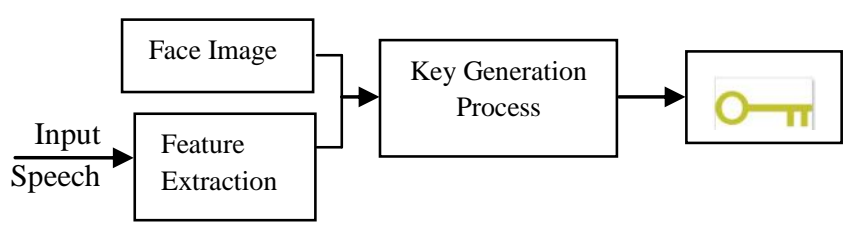

Fig 1. One Time Process of Key Generation during Enrollment

\subsection{Facial Feature Extraction}

Facial recognition has emerged as an attractive solution to address many contemporary needs for human-computer interaction, multimedia management and the verification of identity claims. This might be possible due to the following reasons: the wide range of commercial applications would stand a first place while second is the availability of feasible technologies. It works in a wide range of operating environment from individual home environment to most common public places.

Face recognition is a visual pattern recognition problem. It includes 4 modules - detection, alignment, feature extraction, and matching $[1,20]$.

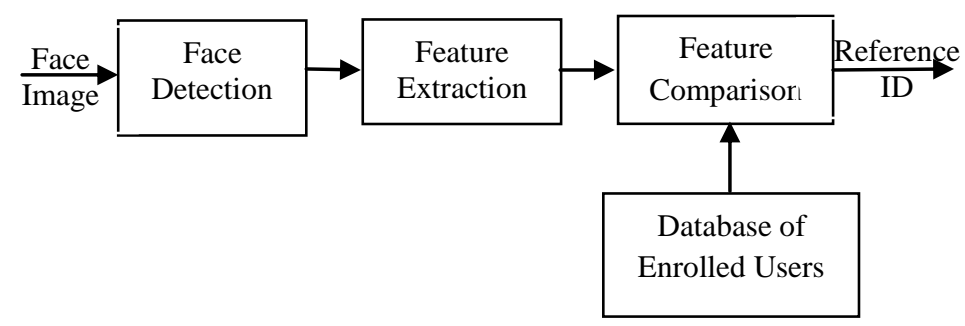

Fig 2. Training of Face Features

\subsubsection{Liveness of the image}

Most of the traditional facial based authentications systems use to capture images straightway. So that it allows the attacker to replay the same face image for authentication. To overcome this problem we added another feature into it called hand gestures $[13,18]$.

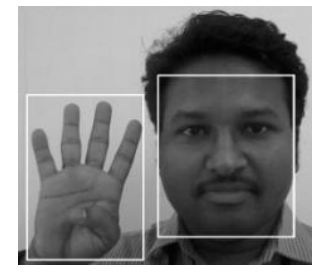

Fig. A.

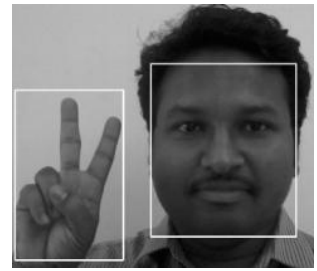

Fig. B.
Dynamically the system asks and captures face with specified Hand gestures during authentication process. Figure 3 depicts the process. This tells the system about liveness of the image. Fig A. and Fig B. shows the separation of image and hand gestures. 


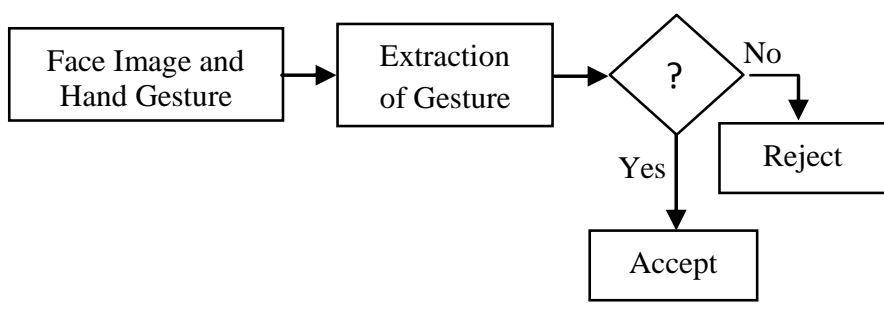

Fig 3. Liveness of the image

$$
\begin{aligned}
& \text { WORK }\left(P_{v}, Q, F\right)=\sum_{i=1}^{m} \sum_{j=1}^{n} d i j \cdot f i j \\
& f i j \geq 0 \text { where } 1 \leq i \leq m \text { and } 1 \leq j \leq n \\
& \sum_{j=1}^{n} \text { fij } \leq \text { wpi where } 1 \leq i \leq \mathrm{m}
\end{aligned}
$$$$
\sum_{j=1}^{m} f i j \leq w q j \text { where } 1 \leq j \leq \mathrm{n}
$$$$
\sum_{i=1}^{m} \sum_{j=1}^{n} f i j=\operatorname{Min}\left(\sum_{i=1}^{m} w p \mid \sum_{j=1}^{n} w q j\right)
$$

Now EMD is :

$E M D(P, Q)=\sum_{i=1}^{m} \sum_{j=1}^{m} d i j \cdot f i j \sum_{i=1}^{m} \sum_{i=1}^{m} f i j$

We calculate the distance between the images and represent it with $\delta$. If $\delta$ is greater than the threshold value we will discard those images if not the image will be considered. After measuring the all images we consider the least distance image.

\subsection{Audio Feature Extraction}

The main purpose of feature extraction is the removal of unnecessary information from the sensor data and conversion of the properties of the signal which are important for the pattern recognition task into a format that simplifies the distinction of the classes. Usually, the feature extraction process reduces the dimension of the data in order to avoid, what is sometimes termed as "the curse of dimensionality" i.e , voice recognition error rates might be reduced and efficiency increased if the "standard" MFCC feature dimension for speech recognition is increased [17, 19]. The goal of the classification step is to estimate the general extension of the classes within feature space from a training set. It had been observed from many previous works that several features are extracted for classifying voice affects such as energy, pitch, formants frequencies, etc.. but notice was dawned that all the above mentioned are prosodic features which are the primary indicators of the respective singer's emotional state. Several studies have shown that MFCC is a good feature for sound classification inclusive of sounds consisting of various mixtures of pitch, frequency, voice etc.. MFCC-based classification also performs well compared to other more complicated features since choosing a proper order of the MFCC feature vector would lead to the successful revelation of the characteristics of a human voice effectively. Therefore, we use MFCC as the short-term feature for classification and calculate it for each frame. A frame is a block of samples within which the signal is assumed to be nearly stationary. MFCC is very much preferred in voice detection since a voice.

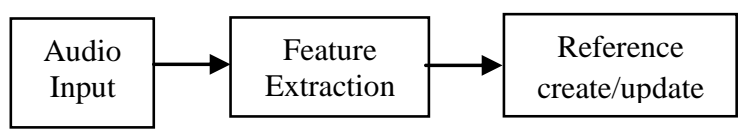

Fig 3. Training of Audio Features 


\section{RESULTS AND ANALYSIS}

The results have been a promising performance note of $80 \%$ accuracy due to their presentation of a harder door to breach the security since the voice and the facial image are combined to a single unit and henceforth requiring the effective use of facial feature and audio extraction and their respectively related algorithms and techniques. Initially the facial image captured for the initial phase of the proposed authentication system. Here the user's submitted facial image is accepted but in order to cross-verify the extent of authenticated user, hand gestures are made to ensure the live display of the image or the verification of the image's live display thereby reducing the chances of forcefully authenticated users from using the respective system and allowing the verification of voluntarily entry to the system which is of huge help to evacuate corruption as well as detect it. As observed in the above diagram showing the various hand gestures and the fact that the hand gestures are presented opposite but in direct alignment with the face specifically helping to show the reality of the user.

Table 1.3 Live Image Test with Hand Gestures.

\begin{tabular}{|c|c|c|c|}
\hline S.No & Gesture & Testing Number & Success Rate(\%) \\
\hline 1 & B & 100 & $96 \%$ \\
\hline 2 & C & 100 & $98.3 \%$ \\
\hline 3 & F & 100 & $97.6 \%$ \\
\hline 4 & O & 100 & 97 \\
\hline 5 & V & 100 & $98.2 \%$ \\
\hline 6 & Y & 100 & $95.3 \%$ \\
\hline
\end{tabular}

In this manner, the live image testing is performed. The live image testing has been proved to be accurate to a satisfying extent as it can be noted from the above given table 1.2 statistics each gesture equal character B,C,F,O,V,Y and its accuracy in terms of percentage.

Followed by the above procedure of submission of face, the use of EMD algorithm ensures the distance $\delta$ between the submitted images to the respective to-be validated images. Now the facial features are extracted from the submitted image using feature extraction mechanism which is validated from the relative or authentication required image in the respective database set. Table 1.3 shows total attempts made to test the user and their success and error rate.

Table 1.4 User Authentication Using Face Recognition

\begin{tabular}{|c|l|c|c|c|}
\hline S.No & $\boldsymbol{\delta}$ & $\begin{array}{c}\text { Total } \\
\text { Attempts }\end{array}$ & $\begin{array}{c}\text { Correct } \\
\text { Alarm }\end{array}$ & $\begin{array}{c}\text { False } \\
\text { Alarm }\end{array}$ \\
\hline 1 & 0.05 & 11 & 10 & 1 \\
\hline 2 & 0.01 & 76 & 74 & 2 \\
\hline 3 & 0.015 & 64 & 63 & 1 \\
\hline 4 & 0.02 & 138 & 137 & 1 \\
\hline
\end{tabular}

\begin{tabular}{|c|c|c|c|c|}
\hline 5 & 0.025 & 126 & 125 & 1 \\
\hline 6 & 0.030 & 185 & 183 & 2 \\
\hline
\end{tabular}

Now the respective user's speech is verified with the system and its features are extracted using audio feature extraction using MFCC approach which acts as a saving substitute for the capture of most of the audio extracted features differentiating them effectively based on their pitch, frequency, voice etc...

Table 1.5 User Authentication Using Audio Feature

\begin{tabular}{|c|c|c|c|c|}
\hline S.No & User & $\begin{array}{c}\text { No Of } \\
\text { Attempts }\end{array}$ & $\begin{array}{c}\text { Correct } \\
\text { Alarm }\end{array}$ & $\begin{array}{c}\text { False } \\
\text { Alarm }\end{array}$ \\
\hline 1 & U1 & 4 & 4 & 0 \\
\hline 2 & U2 & 4 & 3 & 1 \\
\hline 3 & U3 & 4 & 4 & 0 \\
\hline 4 & U4 & 4 & 4 & 0 \\
\hline Total & 4 & 16 & 15 & 1 \\
\hline
\end{tabular}

In the third phase trained voice is authenticated. We tested the voices with 12 filters in MFCC. This kind of a distinguishing makes it easy to recognize one voice from another despite a high amount of similarity. Now the combination of the facial features extracted and extracted audio features into a single units generates a key which is the main authentication formed. Such a combination allows the benefit of saving the permutation of security breach based on facial features and based on audio (i.e) even if audio is mimicked the facial features with gestures can save the security while on the other hand if facial features are manipulated, the respective simultaneous voice requirement can fill in for the security.

\section{CONCLUSION}

The Proposed system is to attempt to resolve a certain amount of security's dimension may be considered as an effective approach due to the efficiency of the algorithms and framework used to build it. In other words, it is technically strong with certain ounces of advantage like checking the liveliness of the image before conforming the facial authentication process and the idea of two level-security through a combination of interdependency with the two factors of voice and facial feature extraction. The security requirement stack is constantly rising with the hits in technology from time to time requiring a dynamic security as a mandatory requirement as observed from the existing systems, that static embedded security amendments are mostly overruled due to their fixative detection by the intent of high technology development. Our proposed system poses a little higher challenge in breaching security with its dynamically enriched combination involving biometric personal identification with gestures for authenticated conformation and voice that together generates the required key in order to use the respective software. 


\section{REFERENCES}

[1] Necmiye Ozayy, Yan Tong Frederick, W. Wheeler and Xiaoming Liu, Improving face recognition with a quality-based probabilistic framework, IEEE Computer Society Conference on Computer Vision and Pattern Recognition Workshops, pp. 134 - 141, 2009.

[2] X. Li, S. Lin, S. Yan, and D. Xu, "Discriminant locally linear embedding with high order tensor data," IEEE Trans. Syst., Man, Cybern. B,Cybern., vol. 38, no. 2, pp. 342-352, Apr. 2008.

[3] Jain, A. K., Nandakumar, K., and Nagar, A. Biometric template security. EURASIP J. Adv. Signal Process 2008 (January 2008), 113:1-113:17.

[4] X. Tan, S. Chen, Z. Zhou, F. Zhang, Face recognition from a single image per person: a survey, Pattern Recognition 39 (2006) 1725-1745.

[5] Wilson, A. (2006). Robust Computer Vision-Based Detection of Pinching for One and Two-Handed Gesture Input. UIST '06. October 15-18, 2006, pp. 255-258.

[6] Sun, D., Li, Q., Liu, T., He, B., and Qiu, Z. A secure multimodal biometric veri_cation scheme. In Advances in Biometric Person Authentication, S. Z. Li, Z. Sun, T. Tan, S. Pankanti, G. Chollet, and D. Zhang, Eds., vol. 3781 of Lecture Notes in Computer Science. Springer Berlin / Heidelberg, 2005, pp. 233-240.

[7] Y.T. Yu, M.F. Lau, "A comparison of MC/DC, MUMCUT and several other coverage criteria for logical decisions", Journal of Systems and Software, 2005, in press.

[8] H. Tan and C. Ngo. "Common Pattern Discovery Using Earth Movers Distance and Local Flow Maximization", IEEE International Conference on Computer Vision, II:1222-1229, 2005.

[9] A. K. Jain, "An introduction to biometric recognition," IEEE Trans. on Circuits and Systems for Video Technology, vol. 14, no. 1, pp. 4-20, January 2004.

[10] P. Indyk and N. Thaper, "Fast Image Retrieval via Embeddings", In 3rd Workshop on Statistical and computational Theories of Vision, Nice, France, 2003.

[11] K.K. Paliwal and B.S. Atal, 'Frequency related representation of speech,' in Proc. EUROSPEECH, p.p.65-68 Sep. (2003).

[12] Forman, G. 2003. An extensive empirical study of feature selection metrics for text classification. J. Mach. Learn. Res. 3 (Mar. 2003), 1289-1305.

[13] Lenman, S., Bretzner, L., Thuresson, B. (2002): Computer vision based hand gesture interfaces for human-computer interaction. Technical Report TRITANA-D0209， 2002， CID-172， Royal Institute of Technology, Sweden.
[14] Y. Rubner and C. Tomasi. Perceptual Metrics for Image Database Navigation. Kluwer Academic Publishers, Boston, MA,2001.

[15] Y. Rubner, C. Tomasi, and L. J. Guibas. "The Earth Mover's Distance as a Metric for Image Retrieval", International Journal of Computer Vision, 40(2):99-121, 2000.

[16] S. Cohen, L. Guibas. "The Earth Mover's Distance under Transformation Sets", IEEE International Conference on Computer Vision, II:1076-1083, 1999.

[17] A. Eriksson and P. Wretling, "How Flexible Is the Human Voice? A case study of mimicry," presented at European Conference Speech Technology, Rhodes, 1997.

[18] J.J. Kuch and T.S. Huang, "Vision-Based Hand Modeling and Tracking," Proc. IEEE Int'l Conf. Computer Vision, Cambridge, Mass., June 1995.

[19] D.A. Reynolds, "Experimental Evaluation of Features for Robust Speaker Identification", IEEE Trans. Speech and Audio Processing2 (4), 1994, 639-643.

[20] R. Brunelli and T. Poggio, "Face recognition: Features versus templates," IEEE Trans. on Pattern Analysis and Machine Intelligence, vol. 15, no. 10, pp. 1042-1052, 1993.

\section{AUTHORS PROFILE}

Srinivas Baggam received M.Tech in (Computer Science \& Engineering) from R.V.R \& J.C college of Engineering, Guntur, Affiliated to Acharya Nagarjuna University. Currently working as an Assistant Professor in M.V.G.R. College of Engineering. He got two and half years of Industrial, two years $R \& D$, Three and half years in teaching Experience.

Neelima Uddavolu received MCA degree and pursuing M.Tech(CN\&IS) from MVGR College of engineering. Her area of interest is network security.

Satheesh Patchikolla received M.Tech in computer science \& science and technology from Andhra university. Currently working as an associate professor in MVGR college of Engineering. He got 1 year industry, 4 years of R\&D and 9 years of teaching experience. his research area is bioinformatics and datamining.

Koduganti Venkata Rao received Ph.D in Computer Science and Engineering from Andhra University, M.Tech in (Computer Science and Technology) from Andhra University and M.Sc (computer science) from Nagarjuna University, 2008, 1999, 1994 respectively. Currently Working as Head of the Department and Professor in Computer Science and Engineering Vignan institute Vishakapatnam. 